\title{
Efeito da vibração de corpo inteiro no paciente hospitalizado: revisão sistemática
}

\author{
Effect of whole body vibration on hospitalized \\ patients: a systematic review
}

\section{Cássio Magalhães da Silva e Silva' \\ Aísa de Santana Lima ${ }^{2}$ Ana Cristina da Costa Oliveira ${ }^{3}$ (1)}

'Autor para correspondência. Universidade Federal da Bahia (Salvador). Bahia, Brasil. cassiofisio2@yahoo.com.br 2-3Universidade Social da Bahia (Salvador). Bahia, Brasil. aisafisio18@gmail.com, fisiocris.16@gmail.com

\begin{abstract}
RESUMO | INTRODUÇÃO: O treinamento com vibração de corpo inteiro (WBV, do inglês Whole Body Vibration) foi recentemente proposto como um método de treinamento com potencial para melhorar a composição corporal e prevenir osteoporose e perda de massa óssea. ${ }^{18}$ Nos últimos anos, alguns estudos mostraram que o WBV pode ser um modo de treinamento benéfico na força, resistência física, atividades relacionadas à mobilidade (transferência, equilíbrio e caminhada) em pacientes com esclerose múltipla ${ }^{19}$, diabetes tipo $2^{20}$, doença pulmonar obstrutiva crôni$c a^{21}$ e receptores de transplante cardíaco.22 Torna-se relevante em razão ao alto impacto na funcionalidade e consequentemente qualidade de vida dos pacientes hospitalizados. OBJETIVO: Verificar o efeito da vibração de corpo inteiro no paciente hospitalizado. MÉTODOS: Revisão de ensaios clínicos controlados randomizados (ECR) e estudo piloto nas bases de dados PubMed, Cochrane Library, Medline e PEDro. As pesquisas nas bases de dados foram realizadas através de combinações (utilizando os conectores "AND" e "OR") através das estratégias de pesquisa PICOS pacientes hospitalizados, vibração de corpo inteiro, fisioterapia, e seus respectivos correlatos em inglês: "hospitalized patients", "whole body vibration", "physiotherapy". Utilizou-se a escala PEDro com o ponto de corte $\geq 5$ para análise da qualidade metodológica. Os critérios de elegibilidade; incluiu pacientes adultos (com idade $\geq 18$ anos); um desenho de ensaio clínico controlado randomizado e estudo piloto; pacientes que utilizaram a vibração de corpo inteiro no âmbito hospitalar. RESULTADOS: Foram incluídos 6 artigos, publicados entre os anos 2014 e 2018, a terapia mostrou-se eficaz em pacientes hospitalizados, havendo significância em alguns desfechos TC6- 167,9 $\pm 117,46 \mathrm{~m}$ para 263,45 $\pm 22124,13 \mathrm{~m} ; \mathrm{p}<0,001$ e VEF1- $32,71 \pm 13,18 \%$ pred. para $3,71 \pm 13,89 \%$, entretanto não houve diferença estatística na PA e FC. CONCLUSÃo: O uso da vibração de corpo inteiro mostrou-se segura e viável em pacientes hospitalizados. O TC6 e o VEF1 apresentado em todos os artigos demonstraram significantes, entretanto não houve diferença estatística na PA e FC. Portanto, é necessário ensaios clínicos randomizados para investigar a eficácia e os efeitos adversos dessa terapia. Embora efeitos positivos tenham sido relatados, sugerimos outras investigações em maior escala com parâmetros controlados e protocolos bem elaborados.
\end{abstract}

PALAVRAS-CHAVE: Vibração do corpo inteiro. Fisioterapia. Pacientes hospitalizados.
ABSTRACT | INTRODUCTION: Whole Body Vibration (WBV) training was recently proposed as a training method with the potential to improve body composition and prevent osteoporosis and bone loss. ${ }^{18}$ In recent years, some studies have shown that WBV can be a beneficial training mode in strength, physical endurance, mobilityrelated activities (transfer, balance, and walking) in patients with multiple sclerosis ${ }^{19}$, type 2 diabetes ${ }^{20}$, chronic obstructive pulmonary disease ${ }^{21}$, and recipients of heart transplantation. $\underline{22}$ It becomes relevant due to the high impact on the functionality and consequently the quality of life of hospitalized patients. OBJECTIVE: To verify the effect of whole-body vibration in hospitalized patients. METHODS: Review randomized controlled clinical trials (RCT) and a pilot study in PubMed, Cochrane Library, Medline, and PEDro databases. The searches in the databases were carried out through combinations (using the "AND" and "OR" connectors) through the search strategies PICOS hospitalized patients, whole-body vibration, physiotherapy, and their respective counterparts in English: "hospitalized patients" "whole-body vibration," "physiotherapy. The PEDro scale with a cutoff point $\geq 5$ was used to analyze the methodological quality. Eligibility criteria; included adult patients (aged $\geq 18$ years); a randomized controlled clinical trial and pilot study design; patients who used whole-body vibration in the hospital setting. RESULTS: Six articles published between 2014 and 2018 were included. The therapy proved to be effective in hospitalized patients, with significance in some outcomes 6MWT- $167.9 \pm 117.46 \mathrm{~m}$ to $263.45 \pm 22124.13 \mathrm{~m} ; \mathrm{p}<0.001$ and FEV1-32.71 $\pm 13.18 \%$ pred. for 3.71 $\pm 13.89 \%$, however, there was no statistical difference in BP and HR. CONCLUSION: The use of whole-body vibration proved safe and viable in hospitalized patients. The 6MWT and FEV1 presented in all articles were significant. However, there was no statistical difference in BP and HR. Therefore, randomized clinical trials are needed to investigate this therapy's efficacy and adverse effects. Although positive effects have been reported, we suggest further investigations with controlled parameters and well-designed protocols on a larger scale.

KEYWORDS: Whole body vibration. Physiotherapy. Hospitalized patients. 


\section{Introdução}

A vibração é um estímulo mecânico oscilatório, caracterizado principalmente por sua frequência e amplitude $^{1,2}$ com possível aplicação clínica, em condições apropriadas, para realização de exercícios em plataformas vibratórias. Nessas plataformas, os indivíduos em ortostase recebem estímulos mecânicos através de seus pés que foram gerados na plataforma. Isso tem sido conhecido como exercício de vibração no corpo inteiro $\mathrm{EVCl} . \underline{3}$

O EVCl é uma importante ferramenta a ser usada em Ciências da Saúde e os efeitos dessa modalidade de exercício têm sido pesquisados em indivíduos saudáveis 4,5 no tratamento de algumas desordens ${ }^{6,7}$ como forma de promoção de condicionamento físico ${ }^{8}$ ou para prevenção e gerenciamento de possíveis problemas clínicos.., 5 Efeitos agudos e crônicos parecem estar associados com essas ações. $\frac{2.9}{9}$

Tal terapêutica tem sido relacionada ao aumento da geração de força nos membros inferiores devido à vibração que induz alterações nos tecidos levando à ativação dos fusos musculares provocando uma contração reflexa para modular a rigidez dos músculos envolvidos. Essa resposta é conhecida como reflexo de vibração tônica. Além disso, as fibras aferentes estimuladas pela vibração do tendão parecem ter efeitos importantes sobre o recrutamento da unidade motora e geração de força. Além disso, o EVCI parece inibir a co-ativação agonista-antagonista através de neurônios inibitórios diminuindo as forças protetoras em torno das articulações ${ }^{1,9,10}$, levando a uma diminuição da força de frenagem ao redor das articulações estimuladas pela vibração. ${ }^{1}$ Sobre os efeitos crônicos, o mecanismo envolvido no EVCl estaria relacionado com as respostas do sistema neuromuscular e hormonal. ${ }^{1}$
No entanto, o uso extensivo de vibrações ainda é contrastante. De fato, mesmo que muitos estudos tenham relatado melhorias notáveis na força muscular após a exposição aguda e crônica à vibração11-14, outros autores não encontraram quaisquer efeitos. $\frac{15,16}{16}$ Uma possível razão para estes resultados de conflito pode ser devido à utilização de diferentes protocolos. De fato, diversos fatores, como tipo de aplicação, amplitude, frequência e tempo de exposição das vibrações, podem ter efeitos agudos, residuais e crônicos sobre o desempenho neuromuscular. ${ }^{17}$

O treinamento com vibração de corpo inteiro (WBV, do inglês Whole Body Vibration) foi recentemente proposto como um método de treinamento com potencial para melhorar a composição corporal e prevenir osteoporose e perda de massa óssea. ${ }^{18}$ Nos últimos anos, alguns estudos mostraram que o WBV pode ser um modo de treinamento benéfico na força, resistência física, atividades relacionadas à mobilidade (transferência, equilíbrio e caminhada) e no humor em pacientes com esclerose múltipla ${ }^{19}$, diabetes tipo $I^{220}$, doença pulmonar obstrutiva crônica ${ }^{21}$ e receptores de transplante cardíaco. 22 Os efeitos do WVB no sistema cardiovascular foram investigados em vários estudos publicados. A diminuição da rigidez arterial após o treinamento com WBV pode reduzir o risco de doença cardiovascular. $.3,24$

O presente estudo torna-se relevante em razão ao alto impacto na funcionalidade e consequentemente qualidade de vida dos pacientes hospitalizados, devido redução de força muscular por consequência do período prolongado de imobilização. Desta forma, este estudo teve como objetivo verificar os efeitos da vibração de corpo inteiro em pacientes hospitalizados na tentativa de melhorar a qualidade de vida dos pacientes, quanto à relação da pressão arterial, frequência cardíaca, volume expiratório forcado no primeiro minuto (VEF1), e o teste caminhada seis minutos, através de um estudo de revisão sistemática. 


\section{Métodos}

O presente estudo trata-se de uma revisão sistemática, no qual foram incluídos e analisados estudos originais, do tipo ensaio clínicos randomizados e estudo piloto, sobre o efeito da vibração de corpo inteiro no paciente hospitalizado. O protocolo da revisão sistemática foi submetido na PROSPERO, número de registro: CRD42020201668. Foi elaborada de acordo com as recomendações da Cochrane Collaboration e relatada de acordo com as diretrizes do Preferred Reporting Items for Systematic Reviews e Meta-Analyzes (PRISMA). ${ }^{25}$

\section{Fontes de dados e estratégias de pesquisa}

A busca dos artigos para obter o desfecho clínico do whole body foi realizada nas bases dados Public Medline (PubMed), Medline e Base de Dados de Evidência Fisioterápica (PEDro) e Scientific Eletronic Library Online (SciELO) até janeiro de 2020 das publicações em língua portuguesa ou inglesa. As pesquisas nas bases de dados foram realizadas através de combinações (utilizando os conectores "AND" e "OR") através das estratégias de pesquisa PICOS pacientes hospitalizados, vibração de corpo inteiro, fisioterapia, e seus respectivos correlatos em inglês: "hospitalized patients" "whole body vibration", "physiotherapy". A estratégia de pesquisa para as bases de dados PubMed consta no Quadro 1.

Quadro 1. Estratégia de pesquisa nas bases de dados PubMed

\section{$\ll<$ Arquivo complementar 1. Estratégia de pesquisa nas bases de dados »»>}

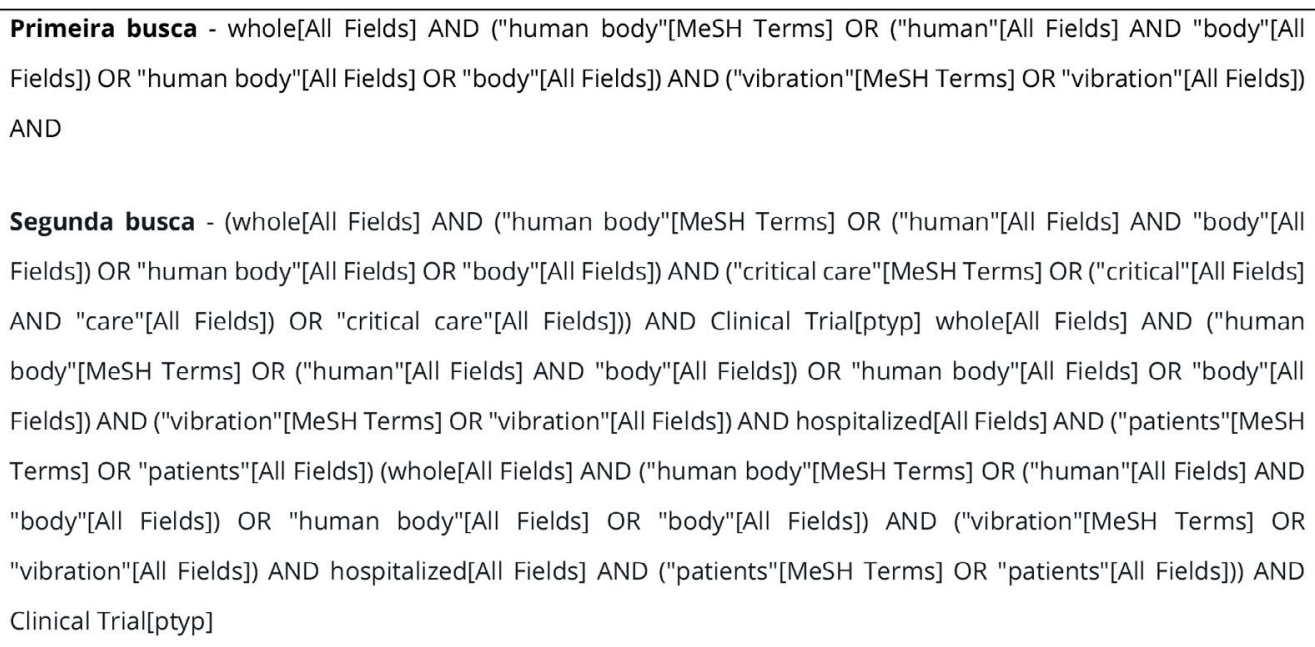

\section{Seleção de estudo}

A seleção dos artigos foi realizada de março de 2019 a janeiro de 2020, sendo elegíveis os que atenderam os seguintes critérios: a) incluiu pacientes adultos (com idade $\geq 18$ anos); b) um desenho de ensaio clínico controlado randomizado e estudo piloto; c) pacientes que utilizaram a vibração de corpo inteiro no âmbito hospitalar. Nenhuma restrição foi feita em termos de sexo, ou a duração da intervenção de exercício. Os artigos foram incluídos pela verificação da coerência entre o título e o objetivo de cada estudo, seguidos da leitura dos resumos. Os principais desfechos de interesse dos artigos selecionados foram: pressão arterial, frequência cardíaca, volume expiratório final (VEF1), teste caminhada de 6 minutos. 


\section{Avaliação da elegibilidade do estudo}

Foram considerados para esta revisão os ensaios clínicos controlados, randomizados e estudo piloto que utilizaram como tratamento a vibração de corpo inteiro no âmbito hospitalar. Foram verificadas as referências de cada artigo selecionado para identificar outros estudos potencialmente qualificados. Foram excluídos os artigos que fornecessem dados insuficientes e aqueles que utilizaram outro treinamento fora do âmbito hospitalar. Após a leitura e a inclusão, os trabalhos foram avaliados para a construção da revisão sistemática.

\section{Qualidade metodológica}

A qualidade dos artigos incluídos foi avaliada utilizando a escala PEDro. Esta avalia os ensaios por meio de 11 itens pré-estabelecidos. O primeiro item é um critério adicional e representa a validade externa (ou "potencial de generalização" ou "aplicabilidade" do estudo clínico), não sendo incluído no escore total da escala. Os demais itens analisam dois aspectos da qualidade do artigo: a validação interna (itens 2 a 9) e se o artigo contém informações estatísticas suficientes para que os resultados possam ser interpretados (itens 10 e 11). Esses itens são qualificados em "aplicável" ou "não aplicável", gerando um escore total que varia entre 0 e 10 pontos.

De forma a buscar um rigor na qualidade metodológica dos artigos selecionados, eles foram analisados e classificados como de "alta qualidade" quando alcançaram escore $\geq 5$ pontos na escala PEDro, ou como de "baixa qualidade" quando obtiveram escore $<4$ na escala referida. Cabe salientar que a pontuação da PEDro não foi utilizada como critério de inclusão ou de exclusão dos artigos, mas sim como um indicador de evidência científica dos estudos, (Tabela 1).

\section{Risco de viés nos estudos primários}

O risco de viés nos estudos incluídos nesta revisão sistemática foi pontuado utilizando o software Review Manager version 5.4 (REVMAN - Cochrane Collaboration), o qual é baseado em domínios, com avaliação crítica feita de forma separada para diferentes aspectos do risco de viés do tipo de estudo em questão. $\frac{26}{}$

\section{Resultados}

\section{Descrição dos estudos selecionados}

A busca inicial realizada nas bases de dados PubMed identificou-se 12 registros, dos quais, 8 foram considerados potencialmente elegíveis quando aplicado o "filtro": ensaio clínico randomizado e estudo piloto, desses 2 artigos foram excluídos por inadequação, após a leitura dos títulos e resumos; sendo assim, 6 artigos preencheram os critérios de elegibilidade e foram incluídos para captação de dados. Toda busca foi iniciada no período de março de 2019. A Figura 1 mostra o diagrama de fluxo PRISMA dos estudos nesta revisão. 


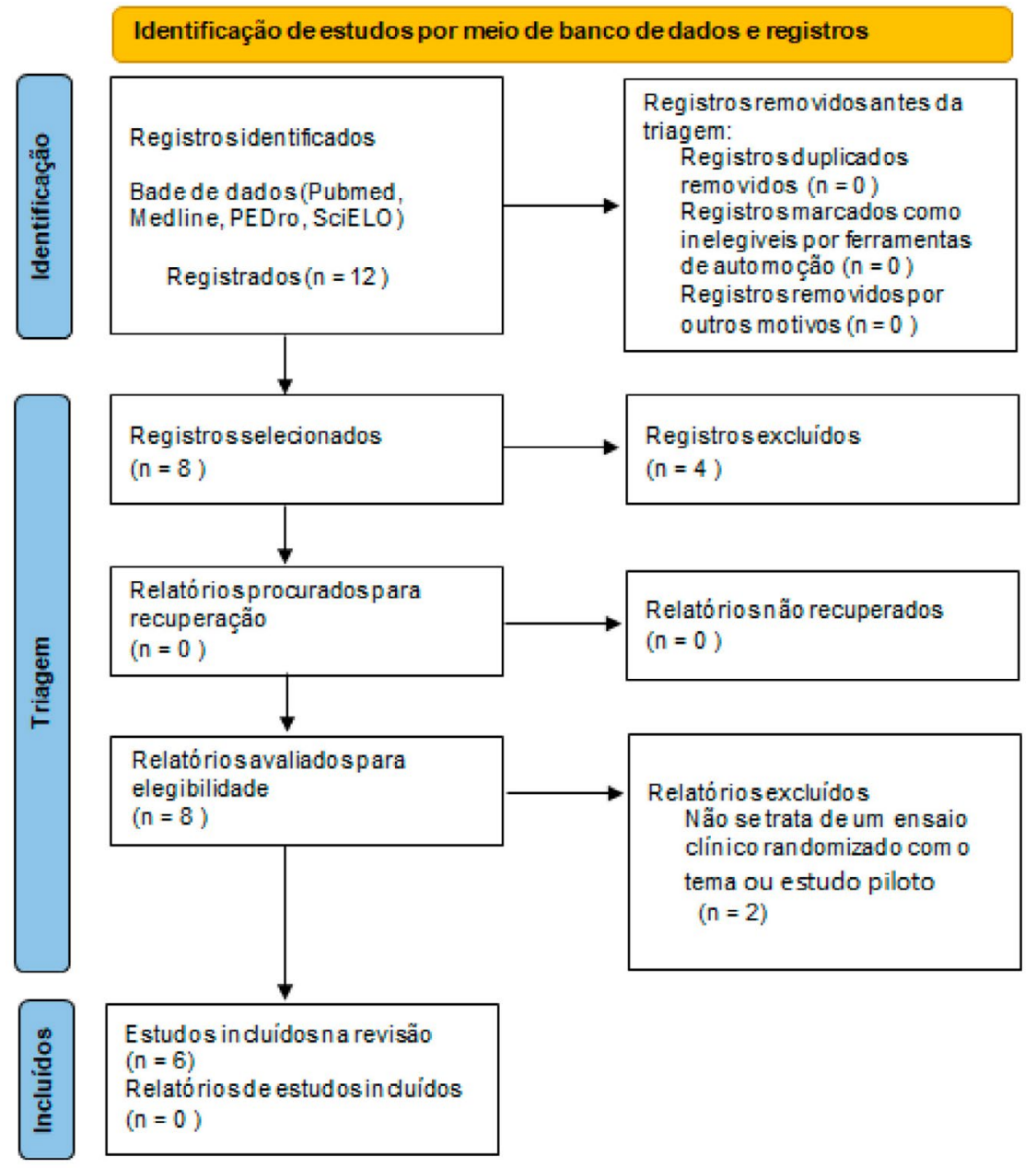

No que se refere à qualidade metodológica dos artigos incluídos (Tabela 1) não houve estudo considerado de "baixa qualidade", uma vez que atingiram pontuação igual ou superior a 5 na Escala PEDro.27

Tabela 1. Qualidade metodológica dos estudos pela Escala PEDro, o uso da vibração de corpo inteiro no paciente hospitalizado, 2019

\begin{tabular}{|c|c|c|c|c|c|c|c|c|c|c|c|c|}
\hline AUTOR & 1 & 2 & 3 & 4 & 5 & 6 & 7 & 8 & 9 & 10 & 11 & TOTAL \\
\hline Greulich et al. $(2014)^{28}$ & $x$ & $x$ & $x$ & $x$ & $x$ & & $x$ & & $x$ & $x$ & $x$ & 8 \\
\hline T. Boeselt et al. (2016) ${ }^{29}$ & $x$ & $x$ & $x$ & & & & & $x$ & $x$ & & $x$ & 5 \\
\hline Brunner et al. (2016) 30 & $x$ & $x$ & & $x$ & $x$ & & & $x$ & & $x$ & $x$ & 6 \\
\hline Wollersheim et al. (2017) ${ }^{31}$ & $x$ & & & $x$ & & & & $x$ & $x$ & $x$ & $x$ & 5 \\
\hline Spielmanns et al. $(2017)^{32}$ & $x$ & $x$ & $x$ & $x$ & $x$ & & $x$ & $x$ & & $x$ & $x$ & 8 \\
\hline Pahl et al. (2018) ${ }^{33}$ & $x$ & $x$ & $x$ & $x$ & $x$ & & $x$ & & $x$ & $x$ & $x$ & 8 \\
\hline
\end{tabular}

Legenda: 1) especificação dos critérios de inclusão (item não pontuado); 2) alocação aleatória; 3) sigilo na alocação; 4) similaridade dos grupos na fase inicial ou basal; 5) mascaramento dos sujeitos; 6) mascaramento do terapeuta; 7) mascaramento do avaliador; 8) medida de pelo menos um desfecho primário em 85\% dos sujeitos alocados; 9) análise da intenção de tratar; 10) comparação entre grupos de pelo menos um desfecho primário e 11) relato de medidas de variabilidade e estimativa dos parâmetros de pelo menos uma variável primária. 


\section{Detalhamento do risco de viés}

Dos sete itens que estão descritos na Cochrane Collaboration, os artigos incluídos nesta revisão foram classificados como baixo risco de viés, (Figuras 2 e 3).

Figura 2. Risco de viés estabelecido pela Cochrane Collaboration $\frac{33}{2}$, dos artigos o uso da vibração de corpo inteiro no paciente hospitalizado, 2019

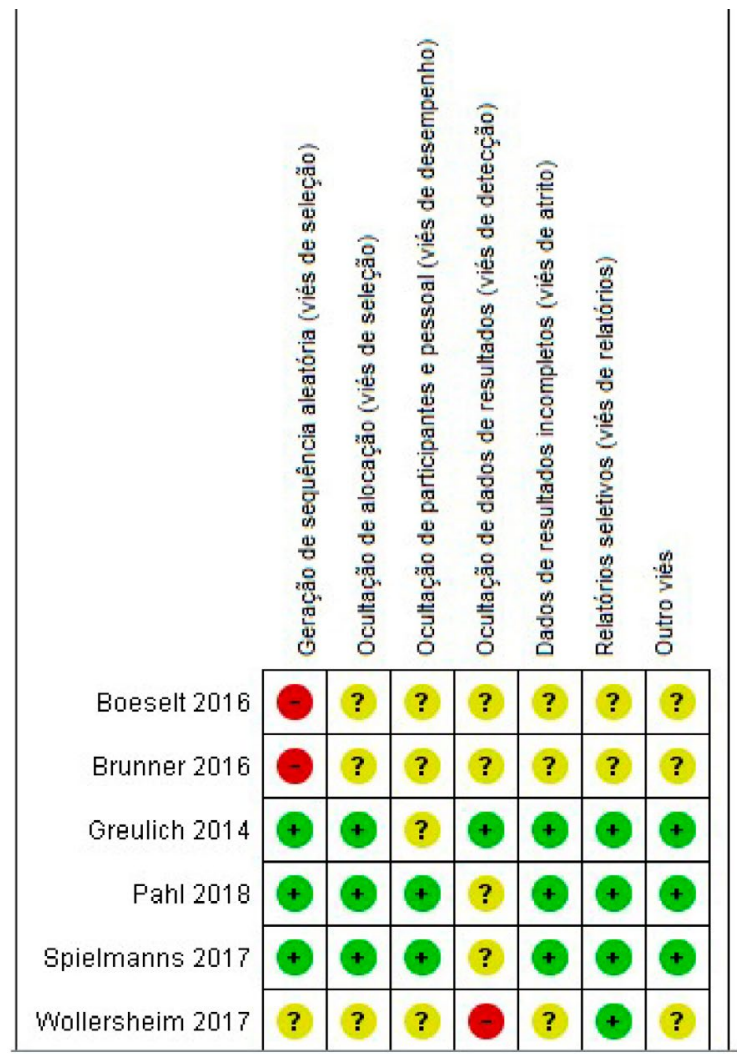

Figura 3. Sumário do risco de viés estabelecido pela Cochrane Collaboration $\underline{33}$, dos artigos o uso da vibração de corpo inteiro no paciente hospitalizado, 2019

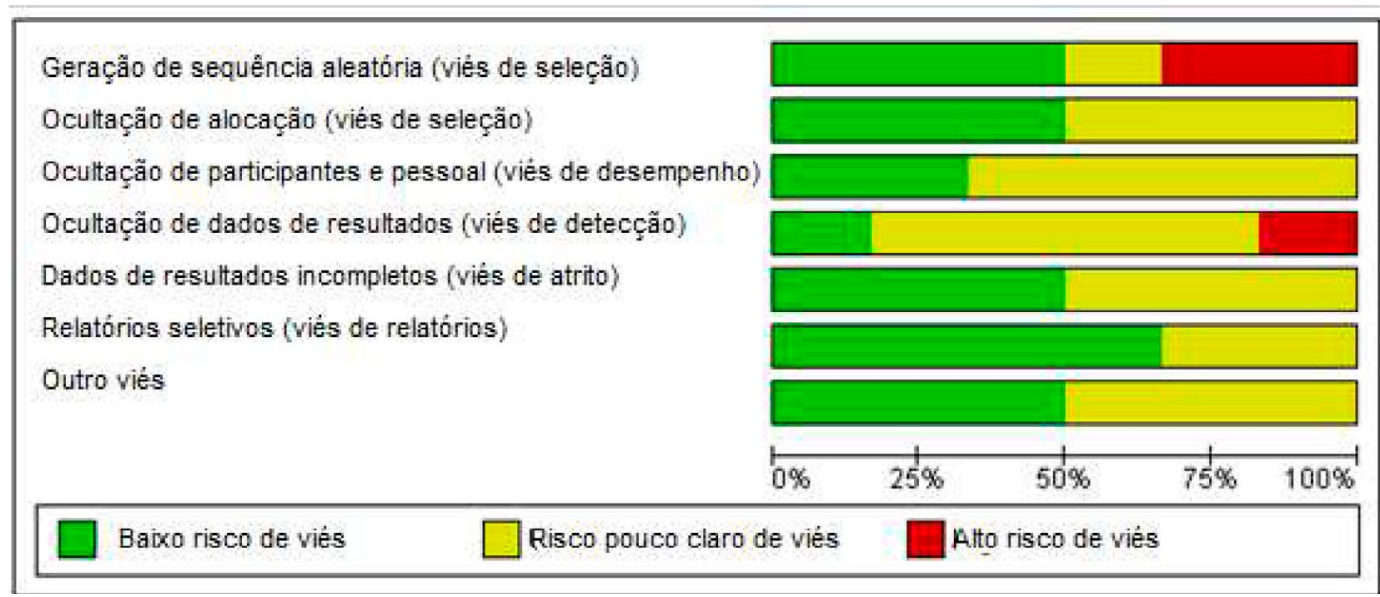

Os artigos incluídos nesta revisão sistemática tiveram ano de publicação entre 2014 e 2018 (tabela 2). O tamanho amostral dos mesmos variou entre 10 e 50 indivíduos adultos, randomizados para o grupo intervenção (Gl) ou grupo controle (GC). As intervenções tiveram duração de no máximo 30 min, e a frequência de 2 a 5 vezes na semana. Através dos resultados foram divididos alguns desfechos considerados importantes: Pressão arterial (PA), Frequência Cardíaca (FC), Volume expiratório final no primeiro segundo (VEF1), e Teste caminhada de 6 minutos (TC6). 
Tabela 2. Características gerais dos estudos incluídos na Revisão Sistemática o uso da vibração de corpo inteiro no paciente hospitalizado, 2019 (continua)

\begin{tabular}{|c|c|c|c|c|c|}
\hline Autor, ano & $\begin{array}{c}\text { Participantes (N - } \\
\text { GI/GC) }\end{array}$ & Intervenção & Desfechos Avaliados & Resultados & Conclusão \\
\hline $\begin{array}{l}\text { Greulich et al. } \\
(2014)^{28}\end{array}$ & $40-20 / 20$ & $\begin{array}{l}\text { Gl= Mobilização + exercícios } \\
\text { respiratórios + movimentos } \\
\text { musculares passivos }+3 \times 2 \text { min/dia de } \\
\text { plataforma vibratória. } \\
\text { GC= } 5 \text { min de mobilização+ } 5 \text { min de } \\
\text { movimentos bruscos } 10 \text { min de } \\
\text { exercícios respiratórios. } \\
\text { A fisioterapia consistiu na } \\
\text { mobilização beira leito, terapia } \\
\text { respiratória e movimentos } \\
\text { musculares passivos. }\end{array}$ & $\begin{array}{l}\text { TC6 } \\
\text { VEF1 } \\
\text { SGRQ } \\
\text { CAT }\end{array}$ & $\begin{array}{l}\text { VEF1 aumentou } \\
\text { significativamente, entre os dois } \\
\text { grupos não houve diferença } \\
\text { significativa. O TC6 houve } \\
\text { melhora significativa no grupo } \\
\text { intervenção, mas não houve no } \\
\text { controle, a diferença entre os } \\
\text { dois grupos foi significativa para } \\
\text { o SGRQ. }\end{array}$ & $\begin{array}{l}\text { A terapia nas exacerbações não } \\
\text { provocou nenhum evento } \\
\text { adverso, induziu benefícios } \\
\text { clinicamente significativos à } \\
\text { capacidade do exercício e a } \\
\text { qualidade de vida. }\end{array}$ \\
\hline T. Boeselt. $\quad(2016)^{29}$ & $24-12 / 12$ & $\begin{array}{l}\text { Posição supina dispositivo preso ao } \\
\text { pé da cama, para transmitir carga } \\
\text { suficiente aos membros inferiores do } \\
\text { paciente leito inclinado } 25^{\circ} \\
\text { GI= Foi realizado em } 2 \text { etapas, placa } \\
\text { no pé vibratória foi realizado o } \\
\text { treinamento sem sapatos por } 3 \text { min } \\
\text { com frequência de } 24 \mathrm{~Hz} \text { e } \\
\text { intensidade média. segunda etapa } \\
\text { após } 1 \text { min de repouso foi adicionada } \\
\text { vibração de corpo inteiro com halter } \\
\text { vibratório. } \\
\text { GC= Vibração de corpo inteiro em } \\
\text { DD+ vibração de corpo inteiro com } \\
\text { halter vibratório. }\end{array}$ & $\begin{array}{l}\mathrm{PA} \\
\mathrm{FC} \\
\mathrm{SpO}_{2}\end{array}$ & $\begin{array}{l}\text { Houve um significativo aumento } \\
\text { da saturação de oxigênio no } \\
\text { sangue }\left(\mathrm{SpO}_{2}\right) \text { no segundo } \\
\text { período (entre o primeiro e } \\
\text { segundo minuto) ( } \mathrm{p}=0,006) \text {. } \\
\text { Para WBVD, diferenças } \\
\text { significativas foram } \\
\text { encontradas somente para } \\
\text { SatO2 e pressão arterial } \\
\text { diastólica no primeiro período } \\
\text { (entre o valor inicial e o } \\
\text { primeiro minuto) ( } \mathrm{p}=0,003) \text {. } \\
\text { Uso exemplar da } \\
\text { eletromiografia (EMG) em um } \\
\text { saudável assunto mostrou } \\
\text { significativamente aumento da } \\
\text { atividade eléctrica do o músculo } \\
\text { quadríceps femoral (vasto } \\
\text { lateral) durante o treinamento. }\end{array}$ & $\begin{array}{l}\text { Não demonstrou nenhum dano } \\
\text { óbvio ao paciente. WBV e a } \\
\text { terapêutica com WBVD devem } \\
\text { ser consideradas para utilização } \\
\text { durante o tratamento na UTI. } \\
\text { São necessários mais estudos } \\
\text { prospectivos randomizados } \\
\text { avaliar os potenciais efeitos } \\
\text { benéficos da WBV e do WBV } \\
\text { terapia na reabilitação em } \\
\text { pacientes de UTI. }\end{array}$ \\
\hline
\end{tabular}


Tabela 2. Características gerais dos estudos incluídos na Revisão Sistemática o uso da vibração de corpo inteiro no paciente hospitalizado, 2019 (continuação)

\begin{tabular}{|c|c|c|c|c|c|}
\hline Autor, ano & $\begin{array}{c}\text { Participantes (N - } \\
\text { GI/GC) }\end{array}$ & Intervenção & Desfechos Avaliados & Resultados & Conclusão \\
\hline Brunner. $(2016)^{30}$ & 10 & $\begin{array}{l}\text { Teve início no } 1^{\circ} \text { dia após alta da UTI, } \\
\text { foi mantido até a transferência para } \\
\text { um centro de reabilitação. Realizado } \\
\text { sob supervisão de um terapeuta } \\
\text { respiratório por } 5 \text { semanas, } 10 \text { min } \\
\text { por dia com frequência até } 10 \mathrm{HZ} \\
\text { GI = } 10 \text { min de vibração de corpo } \\
\text { inteiro. }\end{array}$ & $\begin{array}{l}\text { TC6 } \\
\text { VEF1 } \\
\text { Carga máxima de } \\
\text { trabalho } \\
\text { Pico de fluxo } \\
\text { VC } \\
\text { SF-36 }\end{array}$ & $\begin{array}{l}\text { Todos os desfechos foram } \\
\text { melhorados, porém os que } \\
\text { apresentaram melhora } \\
\text { significativa foram: TC6, VC, } \\
\text { carga máxima de trabalho e } \\
\text { questionário SF-36, entre os } \\
\text { oito aspectos medidos da } \\
\text { saúde, apenas a escala "saúde } \\
\text { médica geral" apresentou uma } \\
\text { melhora significativa após a } \\
\text { conclusão do programa de } \\
\text { treinamento. }\end{array}$ & $\begin{array}{l}\text { WBV é segura e não causou } \\
\text { quaisquer eventos adversos. } \\
\text { treinamento WBV não pode ser } \\
\text { considerado para todos os } \\
\text { pacientes após intervenção, } \\
\text { porque existem várias barreiras } \\
\text { para iniciar esta terapia. Entre } \\
\text { eles, incapacidade para treino } \\
\text { físico, especialmente devido à } \\
\text { doença crítica polineuropatia } \\
\text { após longa permanência na UTI, } \\
\text { tem que seja mencionado. Além } \\
\text { disso, alguns pacientes } \\
\text { subjetivamente sentirem-se } \\
\text { muito fraco ou ansioso por esse } \\
\text { tipo de terapia. }\end{array}$ \\
\hline $\begin{array}{l}\text { Wollersheim. } \\
(2017)^{31}\end{array}$ & 19 & $\begin{array}{l}\text { Fisioterapia passiva única sessão de } \\
\text { vibração de corpo inteiro sessões } \\
\text { durante } 15 \text { min, utilizando } 2 \\
\text { dispositivos, sendo um com vibração } \\
\text { síncrona } 26 \mathrm{HZ} \text { nove vezes para a } \\
\text { frequência de } 1 \text { min e outro com } \\
\text { vibração lateral alternada } 24 \mathrm{HZ} \text { três } \\
\text { vezes durantes } 3 \text { min } \\
\text { Gl= Mobilização durante } 6 \mathrm{~min} \text {, } \\
\text { vibração de corpo inteiro com } \\
\text { duração de } 15 \text { min. }\end{array}$ & $\begin{array}{l}\mathrm{PA} \\
\mathrm{SpO}_{2} \\
\text { Pressão intracraniana } \\
\text { Metabolismo Energético } \\
\mathrm{PH}\end{array}$ & $\begin{array}{l}\text { Os sinais vitais e os parâmetros } \\
\text { hemodinâmicos apresentaram- } \\
\text { se estáveis com mínimas } \\
\text { alteraçõos resultantes da } \\
\text { intervenção, entretanto não } \\
\text { foram consideradas } \\
\text { significantes. }\end{array}$ & $\begin{array}{l}\text { A WBV é segura e viável em } \\
\text { pacientes gravemente } \\
\text { enfermos. } \\
\text { Nossos resultados apoiam o } \\
\text { princípio de que a WBV estimula } \\
\text { o músculo e melhora o } \\
\text { metabolismo muscular, e, } \\
\text { portanto, pode ter o potencial } \\
\text { de prevenir e/ou tratar a } \\
\text { fraqueza de músculo em } \\
\text { pacientes críticos. }\end{array}$ \\
\hline
\end{tabular}


Tabela 2. Características gerais dos estudos incluídos na Revisão Sistemática o uso da vibração de corpo inteiro no paciente hospitalizado, 2019 (conclusão)

\begin{tabular}{|c|c|c|c|c|c|}
\hline Autor, ano & $\begin{array}{c}\text { Participantes (N - } \\
\text { GI/GC) }\end{array}$ & Intervenção & Desfechos Avaliados & Resultados & Conclusão \\
\hline Spielmanns. $(2017)^{32}$ & $27-14 / 13$ & $\begin{array}{l}\text { GI= Vibração lateral } 15 \text { min, } \\
\text { aquecimento } 5 \text { min, treinamento foi } \\
\text { realizado durante } 3 \times 2 \text { min ( } 2 \text { min de } \\
\text { repouso entre eles com frequência de } \\
6-10 \mathrm{HZ} \text { e deslocamento médio pico a } \\
\text { pico de } 4-6 \text { mm durante as primeiras } \\
4 \text { semanas. Durante os intervalos } \\
\text { poderiam sentar-se ou andar } \\
\text { individualmente, a duração do } \\
\text { exercício e frequência da vibração } \\
\text { foram aumentadas progressivamente } \\
\text { ao longo do tempo. Treinamento } 2 x \\
\text { na semana durante } 30 \text { min por } 3 \\
\text { meses. } \\
\text { GC= As sessões durante } 30 \text { min de } \\
\text { relaxamento e reeducação } \\
\text { respiratória em combinação com } \\
\text { exercícios calistênico. }\end{array}$ & $\begin{array}{l}\text { TC6 } \\
\text { SGRQ } \\
\text { CAT }\end{array}$ & $\begin{array}{l}\text { A WBV de baixa intensidade } \\
\text { aumentou o TC6 em 105(45,5- } \\
\text { 133,5) } \mathrm{m}(\mathrm{P}=0,001) \text {, a melhora } \\
\text { entre os grupos foi } \\
\text { significativamente maior } \\
\text { ( } \mathrm{P}=0,001) \text { em relação ao grupo } \\
\text { de tratamento calistênico. } \\
\text { O SGRQ não atingiu } \\
\text { significância, entretanto, a } \\
\text { diferença entre os grupos do } \\
\text { escore do teste de avaliação do } \\
\text { DPOC foi significativamente } \\
\text { maior }(\mathrm{P}=0,02) \text { em favor do } \\
\text { grupo de treinamento } \\
\text { calistênico. }\end{array}$ & $\begin{array}{l}\text { Um programa de WBV de baixo } \\
\text { volume resultou em melhoras } \\
\text { significativas e clinicamente } \\
\text { relevantes na capacidade de } \\
\text { exercício em comparação com } \\
\text { exercícios calistênicos em } \\
\text { indivíduos com DPOC leve a } \\
\text { grave. }\end{array}$ \\
\hline Pahl et al. $(2018)^{33}$ & $11-6 / 5$ & $\begin{array}{l}3 \text { sessões por semana com duração } \\
\text { de } 20 \text { min } \\
\text { GI conjunto de dois a quatro } \\
\text { exercícios diferentes (agachamento } \\
\text { estáticos e dinâmicos, elevação do } \\
\text { calcanhar e combinação de ambos), } \\
\text { cada exercício durou de } 30 \text { a } 60 \text { s, } \\
\text { com repouso de } 30 \text { a } 60 \text { s entre os } \\
\text { exercícios e de } 60 \text { a } 120 \text { s entre } \\
\text { conjuntos a faixa de frequência } 18- \\
25 \mathrm{HZ} \text { e amplitude de } 3,5-4 \mathrm{~mm} \\
\text { GC realizou exercícios aeróbicos em } \\
\text { uma bicicleta ergométrica durante } 20 \\
\text { min os pacientes que não } \\
\text { suportavam tinham um intervalo } \\
\text { individual com períodos de repouso } \\
\text { para cada sessão de exercícios. }\end{array}$ & $\begin{array}{l}\text { PA } \\
\text { FC } \\
\text { Altura do salto } \\
\text { TUG. }\end{array}$ & $\begin{array}{l}\text { Os pacientes do grupo de } \\
\text { intervenção reduziram o tempo } \\
\text { necessário para realizar o teste } \\
\text { TUG }(-1,3 \mathrm{~s}, 95 \% \mathrm{Cl}-2,53-0,7, \\
\mathrm{p}=0,027) \text {, enquanto o grupo } \\
\text { controle não apresentou } \\
\text { alteraçãão }(-1,1 \mathrm{s,95 \%} \mathrm{IC}-6,4-1, \\
\mathrm{p}=0,138) \text {, } \\
\text { A WBV aumentou } \\
\text { significativamente a altura do } \\
\text { salto (2,3 cm, IC } 95 \% \text { 0,1-4,4 } \\
\text { p=0,028), não observaram } \\
\text { alteraçôes arteriais ou da FC } \\
\text { pós exercício em comparação } \\
\text { ao pré-exercício e pós- } \\
\text { intervenção em relação à pré- } \\
\text { intervenão. }\end{array}$ & $\begin{array}{l}\text { Nossos resultados também } \\
\text { sugerem que WBV pode } \\
\text { melhorar a mobilidade e saltar } \\
\text { altura nesses pacientes. Tais } \\
\text { fatores podem estar associados } \\
\text { com maior autonomia e melhor } \\
\text { prognóstico de sobrevida. } \\
\text { Assim, recomendamos a } \\
\text { implementação da WBV como } \\
\text { alternativa método de } \\
\text { treinamento para treinamento } \\
\text { de exercícios aeróbicos durante } \\
\text { quimioterapia intensiva para } \\
\text { manter o status funcional dos } \\
\text { pacientes. }\end{array}$ \\
\hline
\end{tabular}

Legenda: PA: Pressão arterial; FC: Frequência cardiaca; WBV: Whole body vibration (Vibração de corpo inteiro); TC6: Teste de caminhada de 6 minutos; VEF1: Volume Expiratório Forçado n Primeiro segundo; SGRQ: Questionário do Hospital Saint George na Doença Respiratória; CAT: teste de avaliação do DPOC; UTI: Unidade de Terapia Intensiva; VC: Volume corrente; WBVD: Vibração de corpo inteiro com Halter: SF-36: Questionário de Qualidade de Vida: $\mathrm{SPO}_{2}$ : Saturação de oxigênio no sangue: PH: Potencial de hidrogênio. 


\section{Pressão Arterial (PA)}

Ao avaliar a PA três dos seis artigos trouxeram como desfechos sobre a terapia de vibração de corpo inteiro, Pahl et al. $\frac{33}{r}$ relatou que a pressão arterial em paciente hospitalizados durante a quimioterapia quase não houve alteração pelo WBV nem pós exercício em comparação ao pré exercício e pós-intervenção em relação à pré intervenção. Apresentando-se a pressão sistólica no pré-exercício GC-120(115-130) Gl 128 (110-145); pós exercício GC-110 (105-125) GI-110(100123); pré-intervenção GC-114 (112-127) GI-118 (112137); pós-intervenção GC- 119 (111-136) GI 118 (113135); diastólica no pré exercício GC-80(60-85) Gl 80 (70-90); pós exercício GC-75 (70-90) GI-70(70-80); pré intervenção GC-76 (71-82) GI-79(68-86); pós-intervenção GC- 77 (71-84) GI 78 (73-83).

Corroborando com o estudo anterior, Wollersheim et al. $\frac{31}{}$, demonstrou que a pressão arterial diastólica elevou durante o período de Fisioterapia em comparação com a linha de base $p=0,014$ o que não ocorreu durante o WBV, a pressão sistólica não diferiu significativamente da linha de base em nenhum dos momentos de intervenção.

Em contrapartida o estudo Boeselt et al. $\underline{29}$ utilizou pacientes predominantemente pulmonares e cardíacos internados na unidade de terapia intensiva, demonstrou que houve um leve e transitório aumento da pressão arterial diastólica no primeiro período (entre o valor inicial e o primeiro minuto) na intervenção WBVD GC 0.33 (4.25).

\section{Frequência Cardíaca (FC)}

Com relação a $\mathrm{FC}$ dois dos seis artigos analisaram o desfecho Boeselt et al. ${ }^{29}$ demonstrou em seu estudo que ocorreu um discreto aumento reversível em questão de minutos na FC $(P=0,011)$ durante a WBV em pacientes internados na UTI, nenhuma alteração permaneceu após a conclusão da intervenção. $\mathrm{O}$ aumento inicial da FC foi menor nos pacientes em comparação aos controles, o que pode ser devido à influência de drogas que diminuem a FC e a pressão arterial, como os bloqueadores $\beta$ e os inibidores da angiotensina-conversora da enzima (ECA). Para ambas as drogas, uma influência na variabilidade da FC. $\frac{35}{5}$ Entretanto, o estudo de Pahl et al. $\frac{33}{}$, não observou nenhuma alteração nos grupos nos valores da frequência cardíaca pós exercício em comparação ao pré-exercício e pós-intervenção em relação a pré-intervenção.

\section{Volume Expiratório Forçado no Primeiro Segundo (VEF1)}

Com relação a VEF1 dois estudos analisaram o desfecho. Grelich et al. $\stackrel{28}{ }$ demonstrou que durante 0 intervalo de tempo entre admissão e alta hospitalar dos pacientes com exacerbação do DPOC, O VEF1 aumentou significativamente em ambos os grupos, controle: $37,9 \pm 17,41 \%$ para $43,23 \pm 22,8 \% p=0,03$; intervenção $32,71 \pm 13,18 \%$ para $3,71 \pm 13,89 \% p=0,04$ ). Corroborando com o estudo, Brunner et al. .30 demonstrou que o VEF1 foi ligeiramente melhorado em pacientes transplantados de pulmão, mas sem estatística significante (antes da WVB:1,78 $0,14 \mathrm{~L}$; depois: $2,04 \pm 0,18 ; p=0,26$ ).

\section{Teste Caminhada de seis minutos (TC6)}

Ao avaliar a capacidade de exercício através do TC6, Greulich et al. $\frac{28}{}$ explanou que a principal medida de desfecho foi a diferença entre os grupos do teste de caminhada de 6 minutos (dia da alta- dia da internação). Neste mencionado estudo o TC6 aumentou significativamente na WBV, mas não no grupo controle (WBV: $167,9 \pm 117,46 \mathrm{~m}$ para $263,45 \pm 22124,13 \mathrm{~m}$; $p<0,001$ e CON: $203,79 \pm 126,11 \mathrm{~m}$ para 198,67 $\pm 101,37 \mathrm{~m}, \mathrm{p}=0,001$ e $\mathrm{p}=0,001$. A diferença entre o delta de ambos os grupos foi significativa (controle $6,13 \pm 81,65 \mathrm{~m}$ vs WBV 95,55 $\pm 76,29 m ; p=0,007)$.

Brunner et al. .30 , apresentou em seus resultados significância no TC6 após a conclusão do programa (antes:132,3 $\pm 31,2 \mathrm{~m}$; após: $255,5 \pm 43,3 \mathrm{~m} ; \mathrm{p}<0,05)$, o ganho em TC6 foi de $132,2 \pm 20,0 \mathrm{~m}$.

Spielmanns et al. $\frac{32}{}$, observou que o grupo de WBV conseguiram aumentar o TC6 em 105 (45,5-133,5) m $(P=0,001)$, a melhora entre os grupos foi significativamente maior $(P=0,001)$ em relação ao grupo de treinamento calistênico (15[-3 a 21]m, $P=0,10)$.

\section{Discussão}

A presente revisão sistemática verificou que o efeito do uso da vibração de corpo inteiro vem demonstrando melhora significante no que se refere à parte 
funcional com TC6 e pulmonar com VEF1, com efeito semelhante em outros desfechos, porém, não apresentou significância estatística na FC e PA, sendo seguramente aplicável em pacientes hospitalizados.

De acordo com os desfechos TC6 e VEF1 apresentaram significância, em um estudo demonstrou que em pacientes com DPOC, a distância do teste caminhada 6 minutos (DTC6) diminui progressivamente ao longo do tempo, o TC6 contribui para a avaliação completa de pacientes afetados pela doença. ${ }^{36-38}$ Este teste pode ser aplicado em pacientes com insuficiência cardíaca avançada que estejam sendo avaliados para receber transplante de coração. Outros estudos demonstraram que o TC6 é válido e reprodutível em pacientes com insuficiência cardíaca, evidência de que o teste é um meio confiável de avaliar a capacidade funcional em tais pacientes. ${ }^{39}, 40$ Pesquisadores também associaram o pior desempenho no teste de caminhada de pacientes com DPOC com os baixos valores de VEF1, hiper insuflação dinâmica, pior prognóstico da doença (maiores índices de exacerbações) e mortalidade nestes indivíduos. $36,41,42$

Nos desfechos que não houve significância estatística FC e PA, em um experimento conduzido por Robbins et al. $\stackrel{43}{ }$, mostrou um aumento significativo na velocidade do fluxo sanguíneo sem alterações significativas na frequência cardíaca, pressão arterial ou temperatura periférica da pele. $O$ aumento do volume sanguíneo muscular e a velocidade do fluxo sanguíneo após o exercício vibratório foram atribuídos principalmente ao efeito das vibrações na redução da viscosidade do sangue e aumento de sua velocidade através das artérias. ${ }^{44}$ Esses resultados são semelhantes aos encontrados no estudo de Boeselt et al. ${ }^{29}$, que demonstrou que houve um leve aumento na FC. Esses achados indicam que o WBV pode representar uma forma leve de exercícios para o sistema cardiovascular.?

Os artigos Boeselt et al. $\underline{29}$, Wollersheim et al. $\stackrel{31}{\text {, apre- }}$ sentaram alto risco de viés, perfazendo a necessidade de apresentarem informações detalhadas sobre o método utilizado, algo que pode comprometer a viabilidade do estudo. Se esses vieses não forem tratados devidamente, podem repercutir negativamente desde a tomada de decisão em saúde de forma errônea, até a incorporação de novos tratamentos e tecnologias em âmbito nacional que poderão causar mais danos que benefício. $\frac{45}{4}$

Notadamente, a terapia de vibração de corpo inteiro foi bem tolerada pelos pacientes pesquisado nos estudos, no entanto os dados sobre os protocolos da plataforma e seus efeitos nos pacientes necessitam de mais investigações. Estudos maiores são necessários para definir a intensidade e duração ideais da vibração de corpo inteiro, bem como investigar seus possíveis efeitos a longo prazo.

\section{Conclusão}

O uso da vibração de corpo inteiro mostrou-se segura e viável em pacientes hospitalizados. O TC6 e o VEF1 apresentado em todos os artigos demonstraram significantes, entretanto não houve diferença estatística na PA e FC. Portanto, é necessário ensaios clínicos randomizados para investigar a eficácia e os efeitos adversos dessa terapia. Embora efeitos positivos tenham sido relatados, sugerimos outras investigações em maior escala com parâmetros controlados e protocolos bem elaborados.

\section{Contribuições dos autores}

Lima AS participou da concepção, delineamento, busca e análise estatística dos dados da pesquisa, interpretação dos resultados e redação do artigo científico. Silva CMS participou como orientador, pesquisador e revisor, análise estatística dos dados da pesquisa, interpretação dos resultados. Oliveira ACC participou da como pesquisadora, concepção, delineamento, busca redação do artigo científico.

\section{Conflitos de interesses}

Nenhum conflito financeiro, legal ou político envolvendo terceiros (governo, empresas e fundações privadas etc.) foi declarado para nenhum aspecto do trabalho submetido (incluindo, mas não se limitando a subvenções e financiamentos, participação em conselho consultivo, desenho de estudo, preparação de manuscrito, análise estatística etc.). 


\section{Referências}

1. Cardinale M, Bosco C. The use of vibration as an exercise intervention. Exerc Sport Sci Rev. 2003;31:3-7. https://doi. org/10.1097/00003677-200301000-00002

2. Cardinale $M$, Wakeling J. Whole body vibration exercise: are vibrations good for you? Br J Sports Med. 2005;39:585-9. https:// doi.org/10.1136/bjsm.2005.016857

3. Rønnestad BR. Acute effects of various whole-body vibration frequencies on lower-body power in trained and untrained subjects. J Strength Cond Res. 2009;23(4):1309-15. https://doi. org/10.1519/jsc.0b013e318199d720

4. Rittweger J, Just K, Kautzsch K, Reeg P, Felsenberg D. Treatment of chronic lower back pain with lumbar extension and wholebody vibration exercise: a randomized controlled trial. Spine 2002;27(17):1829-34. https://doi.org/10.1097/00007632200209010-00003

5. Roelants M, Delecluse C, Verschueren SM. Whole-bodyvibration training increases knee-extension strength and speed of movement in older women. J Am Geriatr Soc. 2004;52(6):901-8. https://doi.org/10.1111/j.1532-5415.2004.52256.x

6. Connolly DA, Sayers SA, Mchugh MP. Treatment and Prevention of Delayed Onset Muscle Soreness. J Strength Cond Res [Internet]. 2003;17(1):197-208. Disponível em: http://citeseerx.ist.psu.edu/ viewdoc/download?doi=10.1.1.595.7201\&rep=rep1\&type=pdf

7. Rubin C, Recker R, Cullen D, Ryaby J, Mccabe J, Mcleod K. Prevention of postmenopausal bone loss by a lowmagnitude, high-frequency mechanical stimuli: a clinical trial assessing compliance, efficacy, and safety. J Bone Miner Res. 2004;19(3):343-51. https://doi.org/10.1359/JBMR.0301251

8. Delecluse C, Roelants M, Verschueren S. Strength increase after whole-body vibration compared with resistance training. Med Sci Sports Exerc. 2003;35(6):1033-41. https://doi.org/10.1249/01. mss.0000069752.96438.b0

9. Kvorning T, Bagger M, Caserotti P, Madsen K. Effects of vibration and resistance training on neuromuscular and hormonal measures. Eur J Appl Physiol. 2006;96(5):615-25. https://doi. org/10.1007/s00421-006-0139-3

10. Rittweger J, SchiessI H, Felsenberg D. Oxygen uptake during whole-body vibration training exercise: comparison with squatting as a slow voluntary movement. Eur J Appl Physiol. 2001;86(2):16973. https://doi.org/10.1007/s004210100511

11. Issurin VB, Liebermann DG, Tenenbaum G. Effects of vibratory stimulation training on maximal force e flexibility. J Sport Sci. 1994;12(6):561-6. https://doi.org/10.1080/02640419408732206
12. Bosco C, Cardinale M, Tsarpela O, Colli R, Tihanyi J, Duvillard $\mathrm{SP}$, et al. The influence of whole body vibration on jumping performance. Biol Sport [Internet]. 1998;15(3):157-64. Disponível em: https://books.google.com/books?hl=pt-BR\&lr=\&id=PtLm b584Bkc\&oi=fnd\&pg=PA157\&dq=The+influence+on+whole+body+vibration+on+jumping+performance\&ots=HIJ|w4IrMQ\&sig=wgl7o7MGdbVIJyGSNWhxLXMqvfc

13. Cochrane DJ, Stannard SR. Acute whole body vibration training increases vertical jump and flexibility performance in elite female field hockey players. Br J Sport Med. 2005;39:860-5. http://dx.doi. org/10.1136/bjsm.2005.019950

14. Di Giminiani R, Tihanyi J, Safar S, Scrimaglio R. Os efeitos da vibração na força explosiva e reativa ao aplicar frequências de vibração individualizadas. J Sports Sci. 2009;27(2):169-77. https:// doi.org/10.1080/02640410802495344

15. Cochrane DJ, Legg SJ, Hoocker MJ. The Short-Term Effect of Whole-Body Vibration Training on Vertical Jump, Sprint, and Agility Performance. J Strength Cond Res. 2004;18(4):828-32. Citado em: PMID: 15574090

16. Jordan M, Norris S, Smith D, Herzog W. Acute effects of whole-body vibration on peak isometric torque, muscle twitch torque and voluntary muscle activation of the knee extensors. Scand J Med Sci Sports. 2010;20(3):535-40. https://doi.org/10.1111/j. 1600-0838.2009.00973.x

17. Luo J, McNamara B, Moran K. The use of vibration training to enhance muscle strength and power. Sports Med. 2005;35(1):2341. https://doi.org/10.2165/00007256-200535010-00003

18. Gilsanz V, Wren TAL, Sanchez M, Dorey F, Judex S, Rubin C. Low-level, high-frequency mechanical signals enhance musculoskeletal development of young women with low BMD. J Bone Miner Res. 2006;21(9):1464-74. https://doi.org/10.1359/jbmr.060612

19. Santos-Filho SD, Cameron MH, Bernardo-Filho M. Benefits of whole-body vibration with an oscillating platform for people with multiple sclerosis: a systematic review. Mult Scler Int. 2012;2012:274728. https://doi.org/10.1155/2012/274728

20. Behboudi L, Azarbayjani MA, Aghaalinejad H, Salavati M. Effects of aerobic exercise and whole body vibration on glycaemia control in type 2 diabetic males. Asian J Sports Med. 2011;2(2):8390. https://doi.org/10.5812/asjsm.34789

21. Gloeckl R, Heinzelmann I, Kenn K. Whole body vibration training in patients with COPD: a systematic review. Chron Respir Dis. 2015;12(3):212-21. https://doi.org/10.1177/1479972315583049

22. Crevenna R, Fialka-Moser V, Rödler S, Keilani M, Zöch C, Nuhr $M$, et al. Safety of whole-body vibration exercise for heart transplant recipients. Phys Rehab Kur Med [Internet]. 2003;13(5):28690. Disponível em: https://www.thieme-connect.de/products/ ejournals/abstract/10.1055/s-2003-43108 
23. Lai CL, Chen HY, Tseng SY, Liao WC, Liu BT, Lee MC, et al. Effect of whole-body vibration for 3 months on arterial stiffness in the middle-aged and elderly. Clin Interv Aging. 2014;9:821-8. https:// doi.org/10.2147/cia.s60029

24. Figueroa A, Gil R, Wong A, Hooshmand S, Park SV, Vicil F, et al. Whole-body vibration training reduces arterial stiffness, blood pressure and sympathovagal balance in young overweight/ obese women. Hypertens Res. 2012;35(6):667-72. https://doi. org/10.1097/GME.0b013e318294528c

25. Moher D, Liberati A, Tetzlaff J, Altman DG, Altman D, Antes $G$, et al. Preferred reporting items for systematic reviews and meta-analyses: The PRISMA statement (Chinese edition). J Chinese Integr Med. 2009;7(9):889-96. https://doi.org/10.1371/journal. pmed.1000097

26. Carvalho APV, Silva VGA. Bias risk assessment of randomized clinical trials using the Cochrane collaboration tool. Diagnosis Treat [Internet]. 2013;18(1):38-44. Disponível em: http://files.bvs. br/upload/S/1413-9979/2013/v18n1/a3444.pdf

27. Verhagen AP, De Vet HCW, De Bie RA, Kessels AGH, Boers $M$, Bouter LM, et al. The Delphi list: A criteria list for quality assessment of randomized clinical trials for conducting systematic reviews developed by Delphi consensus. J Clin Epidemiol.1998;51(12):1235-41. https://doi.org/10.1016/s08954356(98)00131-0

28. Greulich T, Nell C, Koepke J, Fechtel J, Franke M, Schmeck B, et al. Benefits of full body vibration training in hospitalized patients for COPD exacerbations - a randomized clinical trial. BMC Pulm Med. 2014;14:60. https://doi.org/10.1186/1471-2466-14-60

29. Boeselt T, Nell C, Kehr K, Holland A, Dresel M, Greulich T, et al. Whole- Body Vibration therapy in intensive care patients: A feasibility and safety study. J Rehabil Med. 2016;48(3):316-21. https:// doi.org/10.2340/16501977-2052

30. Brunner S, Brunner D, Winter $H$, Kneidinger N. Feasibility of whole-body vibration as an early inpatient rehabilitation tool after lung transplantation--a pilot study. Clin Transplant. 2016;30(2):938. https://doi.org/10.1111/ctr.12669

31. Wollersheim T, Haas K, Wolf S, Mai K, Spies C, Steinhagen-Thiessen $E$, et al. Whole-body vibration to prevent intensive care unit-acquired weakness: Safety, feasibility, and metabolic response. Crit Care. 2017;21(9). https://doi.org/10.1186/s13054-016-1576-y

32. Spielmanns M, Boeselt T, Gloeckl R, Klutsch A, Fischer $H$, Polanski $H$, et al. Whole-Body Vibration Training Improves Exercise Capacity in Subjects With Mild to Severe COPD. Respir Care. 2017;62(3):3150-23. https://doi.org/10.4187/respcare.05154

33. Pahl A, Wehrle A, Kneis S, Gollhofer A, Bertz H. Feasibility of whole body vibration during intensive chemotherapy in patients with hematological malignancies - a randomized controlled pilot study. BMC Cancer. 2018;18(1):920. https://doi.org/10.1186/ s12885-018-4813-8
34. Sterne JAC, Hernán MA, Reeves BC, Savović J, Berkman ND, Viswanathan M, et al. ROBINS-I: a tool for assessing risk of bias in non-randomized studies of interventions Risk Of Bias In Non-randomized tool for evaluating risk of bias in. Br Med J. 2016;355: i4919. https://doi.org/10.1136/bmj.i4919

35. Pousset F, Copie $X$, Lechat $P$, Jaillon P, Boissel JP, Hetzel M, et al. Effects of bisoprolol on heart rate variability in heart failure. Am J Cardiol. 1996;77(8):612-7. https://doi.org/10.1016/500029149(97)89316-2

36. Pinto-Plata VM, Cote C, Cabral H, Taylor J, Celli BR. The 6-min walk distance: change over time and value as a predictor of survival in severe COPD. Eur Respir J. 2004;23(1):28-33. https://doi.org/ $10.1183 / 09031936.03 .00034603$

37. Torre-Bouscoulet L, Chávez-Plascencia E, Vázquez-García JC, Pérez-Padilla R. Precision and accuracy of "a pocket" pulse oximeter in Mexico City. Rev Invest Clin. 2006;58(1):28-33. Citado em: PMID: 16789596

38. Cote CG, Pinto-Plata V, Kasprzyk K, Dordelly LJ, Celli BR. The 6-min walk distance, peak oxygen uptake, and mortality in COPD. Chest. 2007;132(6):1778-85. https://doi.org/10.1378/chest.07-2050

39. Guimarães GV, Carvalho VO, Bocchi E. Reproducibility of the self-controlled six-minute walking test in heart failure patients. Clinics (Sao Paulo). 2008;63(2):201-6. https://doi.org/10.1590/ \$1807-59322008000200008

40. Ingle L, Shelton RJ, Rigby AS, Nabb S, Clark AL, Cleland JG. The reproducibility and sensitivity of the 6-min walk test in elderly patients with chronic heart failure. Eur Heart J. 2005;26(17):174251. https://doi.org/10.1093/eurheartj/ehi259

41. Spruit MA, Polkey MI, Celli B, Edwards LD, Watkins ML, PintoPlata V, et al. Predicting Outcomes from 6-Minute Walk Distance in Chronic Obstructive Pulmonary Disease. J Am Med Dir Assoc. 2012;13(3):291-7. https://doi.org/10.1016/j.jamda.2011.06.009

42. Cortopassi F, Celli B, Divo M, Pinto-Plata V. Longitudinal changes in handgrip strength, hyperinflation, and 6- minute walk distance in patients with COPD and a control group. Chest. 2015;148(4):986-94. https://doi.org/10.1378/chest.14-2878

43. Robbins D, Yoganathan P, Goss-Sampson M. The influence of whole body vibration on the central and peripheral cardiovascular system. Clin Physiol Funct Imaging. 2014;34(5):364-69. https://doi. org/10.1111/cpf.12103

44. Kerschan-Schindl K, Grampp S, Henk C, Resch H, Preisinger E, Fialka-Moser $\mathrm{V}$, et al. Whole-body vibration exercise leads to alterations in muscle blood volume. Clin Physiol. 2001;21(3):377-82. https://doi.org/10.1046/j.1365-2281.2001.00335.x

45. Carvalho APV, Silva V, Grande AJ. Avaliação do risco de viés de ensaios clínicos randomizados pela ferramentada colaboração Cochrane. Diagn Tratamento [Internet]. 2013;18(1):38-44. Disponível em: http://files.bvs.br/upload/S/1413-9979/2013/v18n1/a3444.pdf 